\title{
Agronegocios
}

\section{Valoración de una finca en periodo de transición a un sistema de producción orgánica en Cipreses de Oreamuno, en la provincia de Cartago, Costa Rica.}

An organic transitional farm valuation, in Cipreses of Oreamuno, Cartago province, Costa Rica.

Nataly Montero-Solís ${ }^{1}$, Carlos González-Blanco², David BarbozaNavarro $^{3}$, Rodrigo Arroyo-Acuña ${ }^{4}$

Fecha de recepción: 07 de setiembre, 2018 Fecha de aprobación: 04 de diciembre, 2018

Montero Solís, N., González Blanco, C., Barboza Navarro, D., \& Arroyo Acuña, R. (2019). Valoración de una finca en periodo de transición a un sistema de producción orgánica en

Cipreses de Oreamuno, en la provincia de Cartago, Costa Rica. Revista EAgronegocios, 5(1). https://doi.org/10.18845/rea.v5i1.4028

Vol. 5. №1. Enero-junio, 2019

DOI: https://doi.org/10.18845/rea.v5i1.4028

${ }^{1}$ Estudiante de la Carrera Acreditada de Economía Agrícola y Agronegocios. Universidad de Costa Rica. Costa Rica. Correo electrónico: naanmoso@gmail.com

2 Estudiante de la Carrera Acreditada de Economía Agrícola y Agronegocios. Universidad de Costa Rica. Costa Rica.

3 Estudiante de la Carrera Acreditada de Economía Agrícola y Agronegocios. Universidad de Costa Rica. Costa Rica.

${ }^{4}$ Estudiante de la Carrera Acreditada de Economía Agrícola y Agronegocios. Universidad de Costa Rica. Costa Rica. 
Resumen

Se procedió a realizar el avalúo de una finca ubicada en Cipreses de Oreamuno, en la provincia de Cartago, Costa Rica, que actualmente se dedica al cultivo de hortalizas y se encuentra en periodo de transición para la producción orgánica. A futuro, se pretende ejecutar un proyecto de inversión en infraestructura, por lo que resulta indispensable conocer el valor actual de la finca y el valor con las mejoras respectivas.

Según la capacidad de uso de la tierra, el terreno se clasifica como $\| \mathrm{Il}_{1} \mathrm{c}_{1}$ con una pendiente de $9 \%$ y se ubica en una zona de Bosque muy Húmedo Premontano. Para realizar el avalúo del terreno se implementaron distintos métodos utilizados en la tasación, incluidos el econométrico, comparativo y financiero; y se realizó un promedio simple de los tres métodos anteriores que permitió estimar el precio $/ \mathrm{m}^{2}$ en $\$ 6$.182, para un valor total del terreno de $\$ 47.392 .188$ con un área de $7.675,75 \mathrm{~m}^{2}$.

También, por medio del método de costeo directo y las tipologías de construcción, respectivamente, se estimó el valor de un invernadero establecido en la finca que asciende a $\$ 9.272 .890$ y la construcción de tres infraestructuras (una cabaña, un granero y un estanque), que corresponden al proyecto de inversión, generando un aumento de $\$ 106.657 .646$ en el valor total de la propiedad, incluyendo mejoras en el servicio eléctrico y en la vía de acceso terrestre.

Palabras clave: Valoración, tasación, beneficio/costo, economía de la tierra.

\section{Abstract}

The appraisal was carried out of a farm located in Cipreses de Oreamuno, in the province of Cartago, Costa Rica, which is currently dedicated to the cultivation of vegetables and is in a transition period for an organic production. In the future, it is intended to execute an infrastructure investment project, so it is essential to know the current value of the farm and the value with the respective improvements.

According to the land use capacity, the land is classified as $\| I e_{1} c_{1}$ with a $9 \%$ slope and it is located in a zone of very Humid Premontane Forest. To perform the land appraisal, different valuation methods were implemented, including the econometric, comparative and the financial method; and a simple average was made of the three previous methods to estimate the price $/ \mathrm{m}^{2}$ in $\mathbb{\|} 6,182$, for a total land value of $\mathbb{4} 47,392,188$ with an area of $7,675.75 \mathrm{~m} 2$.

Also, by means of the direct costing method and construction typologies, respectively, the value of a greenhouse established on the farm amounting $\mathbb{\$} 9,272,890$ and the construction of three infrastructures (a hut, a barn and a pond) were estimated, which correspond to the investment project, generating an increase of $\mathbb{W} 106,657,646$ in the total value of the property, including improvements in the electric service and in the main access road to the farm.

Key words: Valuation, appraisal, benefit/cost, land economy. 


\section{Introducción}

De manera generalizada, se puede decir que la tasación es el precio o avalúo que se asigna a los bienes y/o servicios para un propósito definido, haciendo uso de cálculos económicos, ingenieriles, entre otros (Estela, 2018). Por ello, se realizó el avalúo de una finca en Cipreses de Oreamuno, de la provincia de Cartago, que cuenta con un terreno para uso agrícola de 7.675,75 $\mathrm{m}^{2}$ (metros cuadrados), ubicado a $400 \mathrm{~m}$ de la carretera principal, que se dedicó a la producción de papa y actualmente se encuentra en periodo de transición para la producción orgánica de hortalizas.

Con el fin de conocer la variación en el precio debido a la ejecución de un proyecto de inversión que abarca la construcción de tres tipos de infraestructura, incluidas una cabaña, un granero y un estanque, se realizó un análisis Beneficio/Costo que permitió estimar el cambio en el valor de la propiedad una vez implementado el proyecto de mejora. Esto con el objetivo de facilitar el proceso en la toma de decisiones por parte del dueño de la propiedad.

García (1996) menciona que la agricultura orgánica se caracteriza por ser ecológicamente equilibrada y sostenible, excluyendo el uso de agroquímicos sintéticos para proteger la salud de los trabajadores y consumidores de productos cosechados, y favorecer la recuperación eficiente de las tierras degradadas. Recalca también, el reconocimiento mundial de Costa Rica por su política en conservación de recursos naturales. El avance en la agricultura orgánica en el país ha sido lento pero satisfactorio pues, de acuerdo con Barquero (2016), en el 2015 el área sembrada llegó a 11.055 hectáreas certificadas, $41 \%$ más que en el año anterior, siendo las hortalizas y frutales, después del cacao, los productos que tuvieron un mayor aumento en las hectáreas certificadas, al pasar de 352 en 2014 a 2.118 en 2015.

En Costa Rica, la práctica de avalúos se lleva a cabo principalmente por entidades financieras y/o bancarias cuando se realiza una solicitud crediticia por parte de personas físicas o jurídicas y se requiere de un respaldo hipotecario para reducir el riesgo del crédito; sin embargo, recientemente ha sido objetivo de estudio. Álvarez et al. (2017) realizaron una propuesta integral sobre la valorizaron de una finca rústica en la provincia de Cartago con la implementación de métodos de tasación, en la cual estimaron un precio promedio de $\$ \$ 9,584 / \mathrm{m}^{2}$, mientras que, Paniagua (2015) estimó el valor de indemnización por expropiación basado en el método econométrico para un terreno ubicado en la zona costera del Pacífico Sur, el cual se calculó en \$131.328.

Rosen (1974) propone un modelo de precios hedónicos, definidos como el valor intrínseco de cada una de las características y cantidades de un bien, por lo que el valor total del activo se conforma por la suma total de estos valores. En otros casos a nivel latinoamericano, se han tasado propiedades por medio de la elaboración de multiplicadores, los cuales toman el valor antiguo de la propiedad y la traen a valor presente, representando el efecto de la plusvalía para una región en específico (Carpio, 2014).

\section{Referente teórico}

Según Gujarati y Porter (2010) y Greene (1994), un modelo econométrico es aquel en donde una variable dependiente es explicada por una serie de variables independientes de acuerdo a lo siguiente:

$$
Y=B_{0}+B_{1} X_{1}+B_{2} X_{2}+\cdots B_{k} X_{k}+\hat{u}
$$


Dónde: cada beta corresponde a los coeficientes de regresión para cada una de las variables hasta la k-ésima variable influyente en el modelo y û corresponde a los residuos o errores de cada observación del modelo.

También, una regresión auxiliar consiste en hacer regresiones entre las variables independientes de un modelo econométrico para conocer la respuesta de una variable ante un cambio en otra, midiendo el grado de multicolinealidad del modelo original (Gujarati y Porter, 2010).

El método comparativo según Siniacoff (s.f.) se basa en la comparación de un bien sujeto a estudio de valor respecto a otros bienes ofertados que sean sustitutos testigos, con el fin de deducir el precio más probable que podría alcanzar el bien en análisis. Confirma lo anterior Aznar et al. (2012) al decir que el método comparativo se utiliza para determinar el valor de un bien comparándolo con otros bienes similares de los cuales se conoce su precio por haber sido objeto de una transacción reciente.

Por otro lado, el método financiero resulta de amplia utilización para valorar cualquier bien que produzca ingresos y gastos durante un período determinado (Aznar et al., 2012). Además, según Vargas y Rocha (2017), el precio del terreno se calcula con base a una tasa esperada de retorno que refleja el riesgo de la inversión, por lo que el valor depende de la tasa esperada de rendimiento que los inversionistas exigirían al invertir en la propiedad.

El método de costeo directo para estimar el costo o valor de las infraestructuras, es adecuado debido a que las obras civiles tienden a ser comparadas con sus costos de reemplazo equivalente (Álvarez et al., 2017). Además, Aznar et al. (2012) menciona que al aplicar el método de costo neto de reposición se calcula un valor técnico que se denomina "valor de reposición", que podría ser bruto o neto, el cual debe ser ajustado por el modelo Ross-Heidecke que toma en cuenta el estado de conservación y depreciación de la edificación.

Finalmente, una pendiente es el grado de inclinación o declive que posee un determinado lote con respecto a su eje horizontal (Aznar et al, 2012). Buitrago (2007) indica que la pendiente y el tamaño de un terreno son variables fundamentales para la determinación de su valor, en lo cual coincide Paniagua (2016) pues argumenta que la pendiente influye en la erosión de suelos, y por ende en su valor para generar beneficios.

\section{Metodología}

Utilizando el método econométrico, comparativo y financiero, se debe tomar en cuenta la mayor cantidad de factores que pueden afectar el valor del avalúo. Además, haciendo uso del método de costeo directo y tipologías de construcción, se propone un proyecto de inversión que incluye una cabaña, un granero (barn), un estanque para patos, acometida eléctrica a la finca y una inversión en mejora de la vía de acceso al terreno; estas últimas obtenidas por medio de cotización con empresas especializadas.

Para realizar los modelos econométrico y comparativo se llevó acabo una recopilación de información de terrenos en las inmediaciones, con características similares al avalúo, misma que se respaldó con una busqueda exhaustiva en páginas web; la muestra final es de 24 datos. Para el modelo econométrico, haciendo uso del software Gretl, se aplicaron pruebas para descartar la presencia de heterocedasticidad, autocorrelación y multicolinealidad, y confirmar la normalidad de residuos. Inicialmente se consideraron las siguientes variables explicativas:

- $\quad$ Precio/m $/ \mathrm{m}^{2}(\mathrm{PM})$ : Hace referencia al valor por metro cuadrado de la propiedad. 
- Área (AR): Se refiere a la cantidad de $\mathrm{m}^{2}$ que presenta la propiedad.

- Distancia (DTC): Cantidad de kilómetros (km) que separan el centro de Cartago (localidad cercana con mayor índice de urbanización) con la entrada principal de la propiedad.

- Acceso (ACC): Significa el tipo de vía de acceso a la propiedad.

- Agua (AG): Disponibilidad de agua potable.

- Electricidad (EL): Existencia de sistema eléctrico.

- Uso (US): Donde 1 es de uso agrícola y 2 para quintas.

Con respecto al modelo comparativo, se tomó en cuenta las variables PM, AR, DTC, US y ACC, y se utilizaron los factores de ajuste de la Dirección General de Tributación Directa (DGT) emitidos mediante la Directriz VA-03-2008, y los ajustes obtenidos en el modelo econométrico. Una vez realizados los ajustes respectivos para cada una de las variables en análisis, se procedió a calcular el factor de homologación que permita estimar el precio $/ \mathrm{m}^{2}$ del avalúo.

El método financiero sugiere que, para estimar el precio $/ \mathrm{m}^{2}$ de un terreno de uso agrícola, se necesita conocer el beneficio económico generado mediante el mejor uso. Debido a lo anterior, se realizó un Estado de Flujo Neto de Efectivo para el cultivo de papa, la cual se cultivó en años anteriores en la propiedad. Cabe destacar que los precios utilizados siguen el supuesto de ser el $60 \%$ del precio colocado en CENADA (Paniagua, 2018) y que se realizó una actualización por medio de la tasa de cambio del IPC ${ }^{5}$ de los costos productivos generados por SEPSA (2015).

Para obtener el valor de una cabaña de $96,88 \mathrm{~m}^{2}$, con una combinación de concreto y madera de pino, se utilizaron las tipologías de construcción del MH (2015). También se estimó por medio del método de costeo directo un granero de $200 \mathrm{~m}^{2}$ y un estanque para patos. De la misma manera, se costeó directamente los materiales usados para la construcción de un invernadero de $1.152 \mathrm{~m}^{2}$ y se implementó la metodología de Ross Heidecke para estimar su Valor Neto de Reposición (VNR).

$$
\operatorname{VNR}=\operatorname{VRN}\left(1-\left(\frac{1-\emptyset}{2}\right) *\left(\frac{E}{\mathrm{Vu}}+\frac{\mathrm{E}^{2}}{\mathrm{Vu}^{2}}\right)\right) * \mathrm{C}
$$

Donde,

$$
\begin{array}{ll}
\text { VRN }=\text { Valor de Reposición Nuevo } & \varnothing=\text { porcentaje de recuperación } \\
\mathrm{E}=\text { edad } & \mathrm{C}=\text { estado de conservación } \\
\mathrm{Vu}=\text { vida útil en años } &
\end{array}
$$

\section{Resultados y discusión}

De acuerdo a las características del terreno, el cálculo de la pendiente consistió en dividir el terreno en dos secciones, una con características de planicie y la otra con pendiente más pronunciada. Considerando $6 \operatorname{cotas}^{6}$ por cada sección y su promedio ponderado, el resultado final muestra un $9 \%$ de pendiente.

Para clasificar el terreno según la capacidad de uso de la tierra se consultó el decreto $\mathrm{N}^{\circ} 23214-$ MAG-MINRENEM, resultado una clasificación $\| \mathrm{Ile}_{1} \mathrm{c}_{1}$ (ver anexo 1 ), donde " $\mathrm{e}_{1}$ " describe una

5 Índice de Precios al Consumidor

${ }^{6}$ Nivel de un terreno en metros sobre el nivel del mar (m.s.n.m.). 
pendiente moderadamente ondulada y " $\mathrm{C}_{1}$ " identifica la presencia del terreno en un Bosque muy Húmedo Premontano.

\section{Método econométrico}

Una vez que se realizaron los primeros modelos econométricos para estimar el $\mathrm{precio} / \mathrm{m}^{2}$, se descartaron las variables que no presentaron significancia estadística. Por lo que el valor del terreno se explica por medio de las variables "AR", "DTC" y "US", que resultaron estadísticamente significativas a un nivel de confianza de 95\%. Se realizó transformación logarítmica "log-lin-lin-log" a las variables explicativas para encontrar el modelo de mejor ajuste mediante los criterios de Schwarz y Akaike. El modelo final se muestra en la tabla 1.

Cuadro 1. Modelo econométrico propuesto para estimar el valor del terreno

Variable dependiente: I_PM

\begin{tabular}{|c|c|c|c|c|c|}
\hline & Coeficiente & $\begin{array}{l}\text { Desv. } \\
\text { Típica }\end{array}$ & Estadístico & valor $p$ & \\
\hline const & 10,0946 & 1,03301 & 9,772 & $<0,0001$ & $* *$ \\
\hline DTC & $-0,0297847$ & 0,0129670 & $-2,297$ & 0,0326 & $*$ \\
\hline US & 0,933163 & 0,237456 & 3,930 & 0,0008 & $* *$ \\
\hline I_AR & $-0,221054$ & 0,0851376 & $-2,596$ & 0,0173 & \\
\hline
\end{tabular}

Media de la vble. dep.

D.T. de la vble. dep.

8,788149

0,880567

Suma de cuad.

D.T. de la regresión

Residuos 3,135954

R-cuadrado

0,824160

$F(3,20)$

Log-verosimilitud

31,24665

9,633081

Criterio de Schwarz

31,97838

Rho
0,395977

R-cuadrado corregido

0,797784

Valor $\mathrm{p}$ (de F) $\quad 9,58 \mathrm{e}-08$

Criterio de Akaike

27,26616

Crit. de Hannan-Quinn

28,51631

Durbin-Watson

1,215073

Las variables se interpretan de la siguiente manera: por el aumento de una unidad en la variable DTC, el precio/m² disminuye 2,98\%; por un aumento de una unidad en la variable US, es decir, pasar 
de uso agrícola a quintas, el precio/ $\mathrm{m}^{2}$ aumenta 93,32\%; y, por el aumento de un $1 \%$ en la variable AR, el precio $/ \mathrm{m}^{2}$ disminuye $0,22 \%$.

La función econométrica que explica el valor del terreno se muestra a continuación:

$$
\mathrm{PM}=24.211,4502 \mathrm{e}^{-0,0298 \mathrm{DC}} \mathrm{e}^{0,9332 \mathrm{US}_{\mathrm{AR}}} \mathrm{R}^{-0,2211}
$$

El precio $/ \mathrm{m}^{2}$ obtenido mediante el método econométrico asciende a $\$ 6.051,15$, para un valor total de la propiedad de $\mathbb{4} 46.386 .585$, aproximadamente. Cabe destacar que los coeficientes obtenidos para cada variable explicativa se ajustan a los intervalos de factores de ajuste brindados por la Dirección General de Tributación Directa (DGT) mediante la directriz VA-03-2008. Además, la figura 1 muestra el comportamiento de los precios $/ \mathrm{m}^{2}$ y la predicción respectiva de acuerdo a los coeficientes del modelo econométrico propuesto, en el que se observa un ajuste adecuado entre ambas series.

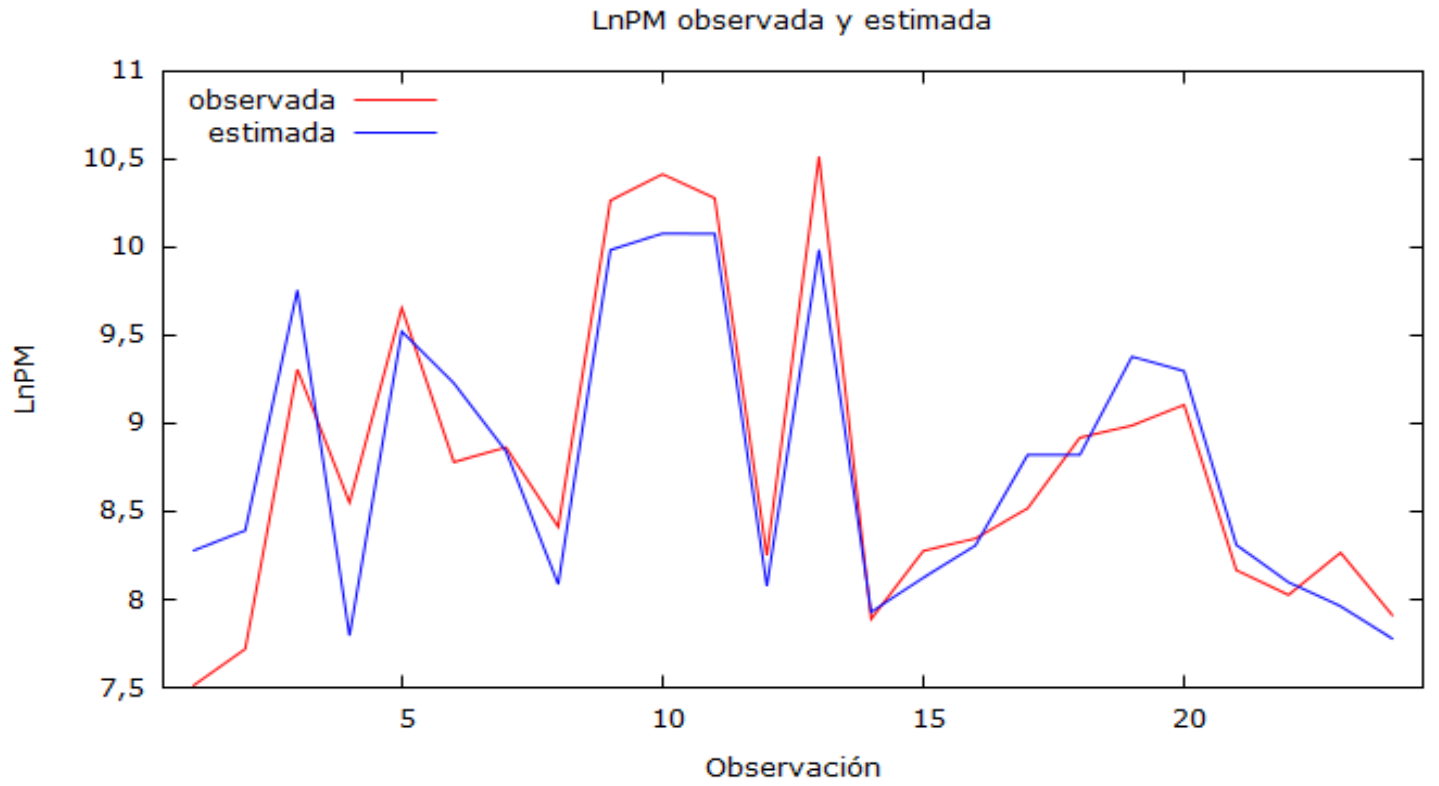

Figura 1. Comportamiento del precio $/ \mathrm{m}^{2}$ observado contra las predicciones del modelo econométrico.

Al modelo final se le realizó un análisis de multicolinealidad mediante el proceso de regresiones auxiliares (ver anexo 2) y se descartó la presencia de la misma. También, se implementó la prueba de Breusch-Pagan para determinar la presencia de heterocedasticidad; el p-Valor obtenido es mayor al 5\% por lo que no se rechaza la Hipótesis Nula ( $\mathrm{HO})$ de "hay homocedasticidad" bajo un nivel de significancia del $5 \%$, por lo que se determina que los errores de las observaciones presentan homocedasticidad (ver anexo 3).

Además, se aplicó la prueba Breusch-Godfrey para determinar la presencia de autocorrelación. El anexo 4 muestra los resultados obtenidos, donde se observa una probabilidad asociada al Multiplicador de Lagrange de 0,476 , por lo que no se rechaza la $\mathrm{HO}$ y se determina que no hay presencia de autocorrelación hasta de orden 3. El resultado se respalda con las características de los datos utilizados pues son de corte transversal (Álvarez et al., 2017).

En lo que respecta a la normalidad de los residuos, se llevó a cabo el contraste de normalidad y no se rechazó la HO, por lo tanto, los residuos siguen una "distribución normal". En la figura 2 se contrasta lo mencionado anteriormente. 
Gráfico Q-Q de uhat1

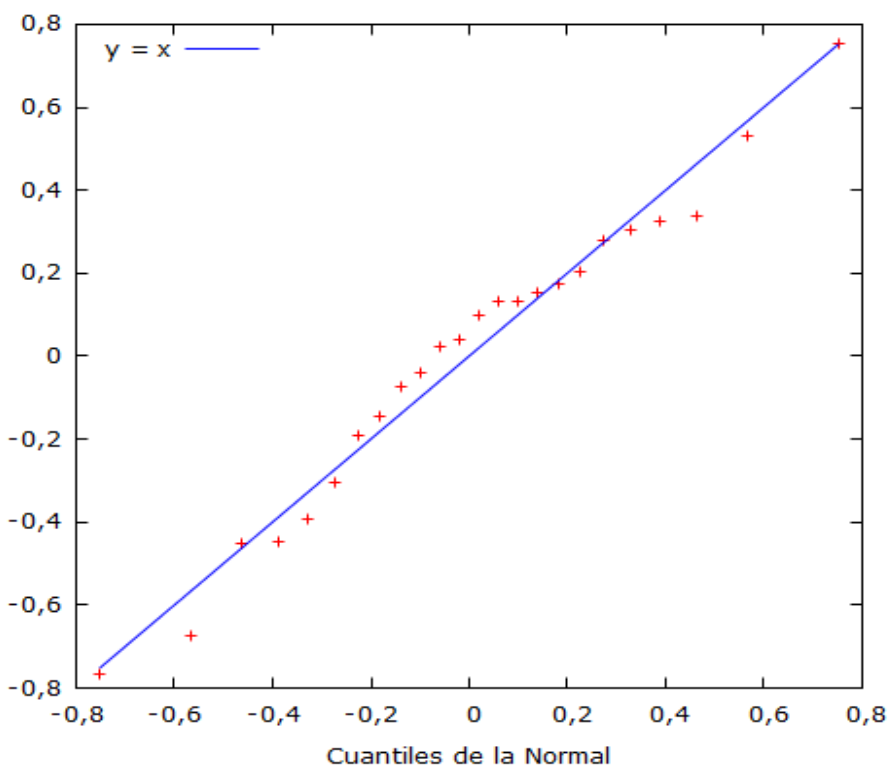

Figura 2. Normalidad de los residuos del modelo econométrico propuesto.

\section{Método comparativo}

En este método se consideraron seis referencias de propiedades con distancias al centro de Cartago menores a $20 \mathrm{~km}$, de uso agrícola o quintas, con áreas entre 1,3 y 18,2 hectáreas y con diferentes condiciones de accesibilidad a la propiedad (ver anexo 5).

Una vez obtenido el factor de homologación por cada referencia, se multiplicó el mismo por el precio/ $/ \mathrm{m}^{2}$ de la propiedad correspondiente, promediando los valores obtenidos para determinar el precio/ $\mathrm{m}^{2}$ del avalúo, el cual se estimó en $\$ 6.498,98$, con una desviación estándar de $\$ 802,82$ y un coeficiente de variación de $12,35 \%$, siendo este mucho menor que el máximo recomendado de $25 \%$ para esta metodología (Paniagua, 2018). El valor total de la propiedad mediante este método se

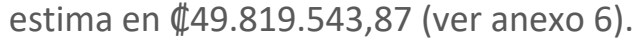

\section{Método financiero}

El ultimo método implementado para estimar el valor del terreno es el financiero, el cual calcula la rentabilidad del alquiler de la tierra dedicada a la producción de papa pues es una actividad agrícola común en la zona de influencia.

El anexo 7 muestra el Estado de Flujo Neto de Efectivo anual para una hectárea en producción de papa, en el que se utiliza un promedio del histórico de precios reales en finca y una producción anual esperada de $26.000 \mathrm{~kg} / \mathrm{ha}$ según criterio de experto (Paniagua, 2018).

El método financiero se puede llevar a cabo de tres maneras y sus resultados se muestran en la tabla 2. El primero da como resultado un precio/ $\mathrm{m}^{2}$ de $\$ 5.997,86$, el segundo de $\$ 3.661,18$ y el tercero de $\mathbb{\$ 7 . 2 1 1 , 5 4}$. Sin embargo, los dos últimos resultados se descartaron pues son incongruentes con el esperado, y el método uno se considera como el más preciso. 
Cuadro 2. Estimación del precio en colones del terreno mediante el método financiero.

\begin{tabular}{|c|c|c|c|c|c|}
\hline \multicolumn{2}{|l|}{ Método 1} & \multicolumn{2}{|l|}{ Método 2} & \multicolumn{2}{|l|}{ Método 3} \\
\hline $\begin{array}{l}\text { Beneficio del } \\
\text { negocio }\end{array}$ & ,83 & $\begin{array}{l}\text { Beneficio } \\
\text { del negocio }\end{array}$ & $\begin{array}{l}1.523 \\
052,42\end{array}$ & \begin{tabular}{|l|}
\multicolumn{2}{|c|}{ Alquiler } \\
anual del \\
terreno
\end{tabular} & $00^{3.000 .000,}$ \\
\hline $\begin{array}{l}\text { Beneficio del } \\
\text { propietario }\end{array}$ &, $23^{2.494 .693}$ & $\begin{array}{l}\text { Perpetuid } \\
\text { ad }\end{array}$ & $\begin{array}{lr} & 1.464 .473,4 \\
8 & \end{array}$ & $\begin{array}{l}\text { Perpetuid } \\
\text { ad }\end{array}$ & 38 2.884.615, \\
\hline Perpetuidad &, $49^{2.398 .743}$ & - & - & - & - \\
\hline Valor total & 7,15 & $\begin{array}{l}\text { Valor } \\
\text { Total }\end{array}$ & 08 36.611.837, & $\begin{array}{l}\text { Valor } \\
\text { total }\end{array}$ &, $62^{72.115 .384}$ \\
\hline Valor/m2 & $5.996,86$ & Valor/m2 & $3.661,18$ & Valor/m2 & $7.211,54$ \\
\hline
\end{tabular}

\section{Estimación del precio/ $/ \mathrm{m}^{2}$ del terreno}

Una vez estimado el precio $/ \mathrm{m}^{2}$ de la propiedad valuada por medio de tres diferentes metodologías (econométrica, comparativa y financiera), se procedió a calcular un promedio simple para obtener el valor definitivo del terreno, el cual es de $\$ 6.182,33 / \mathrm{m}^{2}$, con una desviación estándar de $\$ 225$ y un coeficiente de variación del 3,64\%, para un valor total de $\$ 47.392 .188,89$.

Cuadro 3. Resumen del valor del terreno según metodología y promedio final de la propiedad

\begin{tabular}{lll}
\hline Método & Precio $\left(\mathbb{\$} / \mathrm{m}^{2}\right)$ & Valor total $(\mathbb{W})$ \\
Econométrico & $6.051,15$ & $46.386 .603,11$ \\
Financiero & $5.996,86$ & $45.970 .419,70$ \\
Comparativo & $6.498,98$ & $49.819 .543,87$ \\
Promedio & $6.182,33$ & $47.392 .188,89$ \\
\hline
\end{tabular}

\section{Valoración de la infraestructura actual}

En la propiedad se encuentra un invernadero construido en la misma finca, el cual cuenta con dimensiones de $24 \times 48 \mathrm{~m}$ (ancho $\mathrm{x}$ largo). El invernadero cuenta a lo ancho con 4 túneles de $6 \mathrm{~m}$ de ancho cada uno y, a lo largo, postes de soporte cada $3 \mathrm{~m}$; el techo es de cubierta plástica "dripblock", los laterales son de malla anti-insecto $40 / 25$ y los soportes en tubo redondo galvanizado de diferentes diámetros. Debido a la especificidad del invernadero y la dificultad de compararlo con uno similar, se procedió a valorarlo por el método de costeo directo (ver anexo 8) y se obtuvo un VRN de $\$ 10.326 .068\left(\$ 8.964,60 / \mathrm{m}^{2}\right)$.

La infraestructura tiene 8 años de edad, su vida útil es de 50 años, presenta un estado de conservación "bueno" y al finalizar su vida útil se podrá recuperar un $15 \%$ de su valor mediante la venta de los tubos metálicos. Se aplicó el método Ross-Heidecke para obtener el VNR, el cual se estima en $\$ 8.049,38 / \mathrm{m}^{2}$ y en $\$ \$ 9.272 .890$ el valor total del invernadero. 
De acuerdo con los resultados obtenidos con los métodos econométrico, comparativo y financiero, al sumar el valor del invernadero el valor total de la finca asciende a $\$ 56.665 .079$.

\section{Valoración de la infraestructura del proyecto de mejora}

Se costeó de manera directa la construcción de un granero de $200 \mathrm{~m}^{2}$ (ver anexo 10), de dos niveles que permita establecer un sistema estabulado para 20 ovejas destinadas a la producción de leche, tres establos para caballos y una sección para carga y descarga de insumos; todo lo anterior en el primer nivel. El segundo nivel no tendrá divisiones y se destinará al almacén de insumos y herramientas agrícolas. Adjunto a esto se valoró la construcción de un estanque para patos para cumplir con un objetivo paisajista; éste será de $63 \mathrm{~m}^{3}$. El valor total de estas dos infraestructuras es de $\$ 33.292 .664,25$.

La cabaña cumple con los siguientes acabados: paredes con zócalo de concreto y madera de pino, de 1,2 y 1,8 m de altura respectivamente, estructura de columnas de concreto en las esquinas y una viga solera completa alrededor de la cabaña para dar soporte al techo, que será estilo abovedado con cerchas de madera expuestas, pisos de cerámica, amplios ventanales y techos en teja tipo Toledo (ver anexo 9). El precio de la cabaña se estimó de acuerdo a la tipología CÑO2 de $\mathrm{MH}$ (2015), el cual es de $\$ 336.293,56 / \mathrm{m}^{2}$ una vez actualizado según el IPC cons $^{7}$. La cabaña mide 96,88 $\mathrm{m}^{2}$ y el costo total es de $\$ 32.580 .065,80$, al cual se le agregó un $15 \%$ sobre el valor para considerar la construcción de una chimenea y un corredor estilo porche, dando un valor final de $\$ 37.467 .075,70$.

En lo que respecta al establecimiento del servicio eléctrico se debe considerar la instalación del sistema desde la carretera principal hasta la finca, recorriendo una distancia de $400 \mathrm{~m}$. Existen tres opciones para facilitar el acceso al servicio eléctrico: a) por medio de cableado que incluye la instalación de un booster eléctrico, que será el sistema a implementar, con un costo $\$ 7.000 .000 ;$ b) por medio de cableado y un transformador eléctrico por un monto de $\$ 8.500 .000$ (Castro, 2018); y, c) la instalación de paneles solares, los cuales tiene un costo de $\$ 5.600 .000$ que requieren un mantenimiento de $\$ 350$ anuales (Paneles Solares Purasol, 2018).

Finalmente, para la mejora de la vía terrestre de acceso a la finca se cotizó con la empresa especializada Stratega Solution (2018) que, considerando las características de la zona y la estabilidad del suelo, estimó un costo de $\$ 19.000 .000$ que incluye remover el material de desecho del camino reubicándolo en la misma finca, una capa de $25 \mathrm{~cm}$ de lastre, corta de materia vegetal, $3 \mathrm{~m}$ de ancho y un lindero de cunetas de $75 \mathrm{~cm}$ en cada costado por una longitud de $500 \mathrm{~m}$.

\section{Análisis Beneficio/Costo del proyecto de mejora}

Con el fin de determinar el efecto del proyecto inversión sobre la mejora de la finca respecto a su valor económico, el método comparativo permite considerar la inversión en la vía de acceso al terreno y servicio eléctrico, homologando el valor respecto a la finca en el estado actual (referencia 1), obteniendo un nuevo precio/ $\mathrm{m}^{2}$ de $\$ \$ 9.655,57$ y un total de $\$ 74.017 .205$ para el valor de la finca.

Cuadro 4. Método comparativo para la finca con las mejoras vía de acceso terrestre y servicio de electricidad.

7 Índice de Precios de la Construcción. 


\begin{tabular}{|c|c|c|c|c|c|}
\hline Variables & Acceso & $\begin{array}{l}\text { Servicio } \\
\text { Públicos }\end{array}$ & 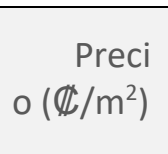 & $\begin{array}{l}\text { Factor de } \\
\text { homologació } \\
\text { n }\end{array}$ & \begin{tabular}{l}
\multicolumn{1}{c}{ Valor } \\
homologado \\
$\left(\mathbb{\&} / \mathrm{m}^{2}\right)$
\end{tabular} \\
\hline $\begin{array}{l}\text { Tipo de } \\
\text { función }\end{array}$ & Exponencia & Exponencia & - & - & - \\
\hline Avalúo & 5 & 11 & - & - & - \\
\hline a 1 Referenci & 9 & 5 & 6.182 & - & - \\
\hline $\begin{array}{r}\text { Factor de } \\
\text { homologación }\end{array}$ & 1,30 & 1,20 & - & 1,56 & 9.656 \\
\hline & & & \multicolumn{2}{|c|}{ 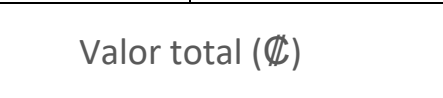 } & $\begin{array}{ll} & 74.017 .20 \\
5 & \end{array}$ \\
\hline
\end{tabular}

Una vez determinado el nuevo valor de la propiedad se estimó la viabilidad de ese proyecto al comparar el beneficio estimado en el aumento en el valor de la finca entre los costos de estas mejoras mediante la relación Beneficio/Costo, tomando en cuenta los $\mathbb{\$ 7 . 0 0 0 . 0 0 0 ~ d e ~ l a ~ a c o m e t i d a ~}$ eléctrica y los $\$ 19.000 .000$ por el arreglo del camino, cálculo que se presenta a continuación:

$$
\mathrm{B} / \mathrm{C}=\frac{\not 26.625 .009}{\not 26.000 .000}=1,0240
$$

Los costos de las mejoras son menores que el aumento en el valor de la propiedad valuada debido a que el coeficiente es mayor a 1, por lo tanto, se recomienda llevar a cabo la inversión. No obstante, la opción puede ser más factible si este costo es asumido entre todos los vecinos que se verían beneficiados por el acceso al servicio eléctrico y la mejora en la vía de acceso terrestre.

Finalmente, una vez implementado el proyecto de inversión en la finca ubicada en Cipreses de Oreamuno, su valor ascendería a $\$ 154.049 .835, \$ 97.384 .756$ más que el valor actual asumiendo únicamente el terreno y el invernadero, lo que representa un aumento de $172 \%$ en el valor de la propiedad. Sin embargo, es necesario hacer un análisis financiero y de Beneficio/Costo para determinar la rentabilidad futura del proyecto, tomando en cuenta los ingresos económicos que se obtendrían por la producción y venta de hortalizas, leche de origen ovino, entre otras actividades económicas que se puedan desarrollar. Además, resulta importante considerar el poder de negociación con los vecinos para establecer el servicio eléctrico y la mejora de la vía de acceso a la propiedad valuada y propiedades aledañas de manera conjunta, disminuyendo el monto de la inversión por persona involucrada y generando un mayor impacto económico y social.

\section{Literatura citada}

Álvarez, D., Gómez, K., Madriz, D., Méndez, C. y Murillo, A. (2017). Valoración de la finca rustica "La Laguna" en Tierra Blanca, provincia de Cartago: Una Propuesta Integral. e-Agronegocios. 3 (2), 1$13 . \quad$ Recuperado de https://docs.google.com/viewer?a=v\&pid=sites\&srcid=ZGVmYXVsdGRvbWFpbnxlYWdyb25IZ29ja W9zdWNyfGd4Ojc0NWM2OTY4YWUXMWVjYZY. 
Aznar, J., González, R., Guijarro, F. y López, A. (2012). Valoración inmobiliaria. Métodos y aplicaciones. [Versión digital]. Valencia: Universitat Politècnica de València. Recuperado de https://riunet.upv.es/bitstream/handle/10251/19177/Valoraci\%C3\%B3n\%20inmobiliaria.pdf?sequ ence $=1$.

Barquero, M. (2016, 05 de julio). Terrenos orgánicos en Costa Rica repuntaron en dos últimos años. La Nación. Recuperado de https://www.nacion.com/economia/agro/terrenos-organicos-en-costarica-repuntaron-en-dos-ultimos-anos/6652KW7OSJBOZFCGANEUYMNQVQ/story/.

Buitrago, O. (2007). Factores determinantes del precio de la tierra rural en los municipios contiguos a Bogotá D.C. Cali: Universidad del Valle. 177 p.

Castro, M. (2018, 04 de julio). Cotización presencial de sistema eléctrico. San José, Costa Rica.

Carpio, D. (2014). Precio del suelo y metodologías de avaluación. Exploración para la captura de plusvalías urbanas en el caso de la región metropolitana de Santiago de Chile. Santiago de Chile: Universidad de Chile. Recuperado de: http://repositorio.uchile.cl/bitstream/handle/2250/130467/precio-del-suelo-y-metodologias-de$\% 20$ avaluacion.pdf?sequence $=1$

Decreto $n^{\circ}$ 23214-MAG-MINRENEM. (1994, 06 de junio). Metodología para la determinación de la capacidad de uso de las tierras de Costa Rica. Diario La Gaceta. Costa Rica.

DGT (Dirección General de Tributación Directa). (2008). Directriz VA-03-2008. San José, Costa Rica.

Doomos. (2018). Bienes raíces en Costa Rica: Quintas, lotes, fincas, terrenos. Recuperado de http://www.doomos.co.cr/.

Encuentra24. (2018). Bienes raíces en Costa Rica: Fincas. Recuperado de https://www.encuentra24.com/costa-rica-es/bienes-raices.

Estela, N. (2018). Nuevos criterios de tasación de inmuebles. Scribd. Recuperado de https://es.scribd.com/doc/193953001/Nuevos-Criterios-de-Tasacion-de-Inmuebles-PDF.

García, J. (1996). Agricultura Orgánica en Costa Rica. San José: MAG. Recuperado de http://www.mag.go.cr/congreso_agronomico_x/a50-2388-I_001.pdf.

Greene, W.H. (1997). Econometric analysis. New Jersey: Prentice-Hall.

Gujarati, D. y Porter, D. (2010). Econometría. (5. ${ }^{a}$ ed.). México: MacGraw-Hill Interamericana.

InmoTico. (2018). Bienes raíces en Costa Rica: Fincas. Recuperado de http://www.inmotico.com/.

MH (Ministerio de Hacienda). (2015). Manual de valores base unitarios por tipología constructiva. San José, Costa Rica. Recuperado de http://www.hacienda.go.cr/docs/544815600c587_MVBUTC_2015V3.pdf.

OLX. (2018). Bienes raíces en Costa Rica: Fincas. Recuperado de https://www.olx.co.cr/lotesterrenos-cat- 410 .

Paneles Solares Purasol. (2018, 06 de julio). Cotización telefónica de paneles solares y cálculo de energía solar. San José, Costa Rica. Recuperado de https://bacsolar.cr/calculadora.

Paniagua, J. (2015). Estimación de valor de indemnización por expropiación para un terreno ubicado en la zona costera del Pacífico Sur, con base en el método econométrico de valuación. e$\begin{array}{lllll}\text { Agronegocios. } & 2 & (1), & 11 . & \text { Recuperado de }\end{array}$ https://sites.google.com/site/eagronegociosucr/numeros-anteriores. 
Paniagua, J. (2016). Estimación del efecto de la pendiente sobre el valor de la tierra en la zona de Damita, Cañón del Guarco, por medio del método econométrico de valuación. e-Agronegocios. 2 (2), 1-13. Recuperado de https://sites.google.com/site/eagronegociosucr/numeros-anteriores.

Paniagua, J. (2017). Efecto de la distancia a centro urbano sobre el valor de las tierras rurales: El caso de Guápiles. e-Agronegocios. 3 (1), 1-11. Recuperado de https://sites.google.com/site/eagronegociosucr/numeros-anteriores.

Paniagua, J. (2018, 05 de julio). Entrevista personal sobre metodologías de tasación y criterio de experto. Universidad de Costa Rica, San José, Costa Rica.

Rosen, S. (1974). Hedonic prices and implicit markets: Product differentiation in pure competition. 82 (1), 34-55. Recuperado de: https://pdfs.semanticscholar.org/c66b/420f81caa64ad7a8e1cce2fd6ea7218099ff.pdf

SEPSA (Secretaría Ejecutiva de Planificación Sectorial Agropecuaria). (2015). Costeo para la producción de papa. San José, Costa Rica. Recuperado de http://www.infoagro.go.cr/EstadisticasAgropecuarias/Documents/COSTO_PRODUCCION/COSTO_ PRODUCCION_papa_2015.pdf.

SEPSA (Secretaría Ejecutiva de Planificación Sectorial Agropecuaria). (2018). Precios históricos de papa. San José, Costa Rica. Recuperado de http://www.infoagro.go.cr/BEA/BEA28/precios.html.

Siniacoff, M. (s.f.). Método comparativo de mercado. Uruguay: ProEVA. Recuperado de https://eva.fing.edu.uy/pluginfile.php/105308/mod_folder/content/0/M\%C3\%89TODO\%20COMP ARATIVO\%20DE\%20MERCADO.pdf?forcedownload=1.

Stratega Solution. (2018). Cotización telefónica de mejora de caminos con Fabián Estrate. Cartago, Costa Rica.

Vargas, A. y Rocha, J. (2017). Valoración de inmuebles comerciales mediante la aplicación del método de capitalización directa y el método de flujos de caja descontados. Investigación y

Desarrollo. $16 \quad$ (2), 87-104. Recuperado de http://www.scielo.org.bo/scielo.php?script=sci_arttext\&pid=S2518-44312016000200007.

\section{Anexos}

Anexo 1. Clasificación del terreno de acuerdo al uso de la tierra.

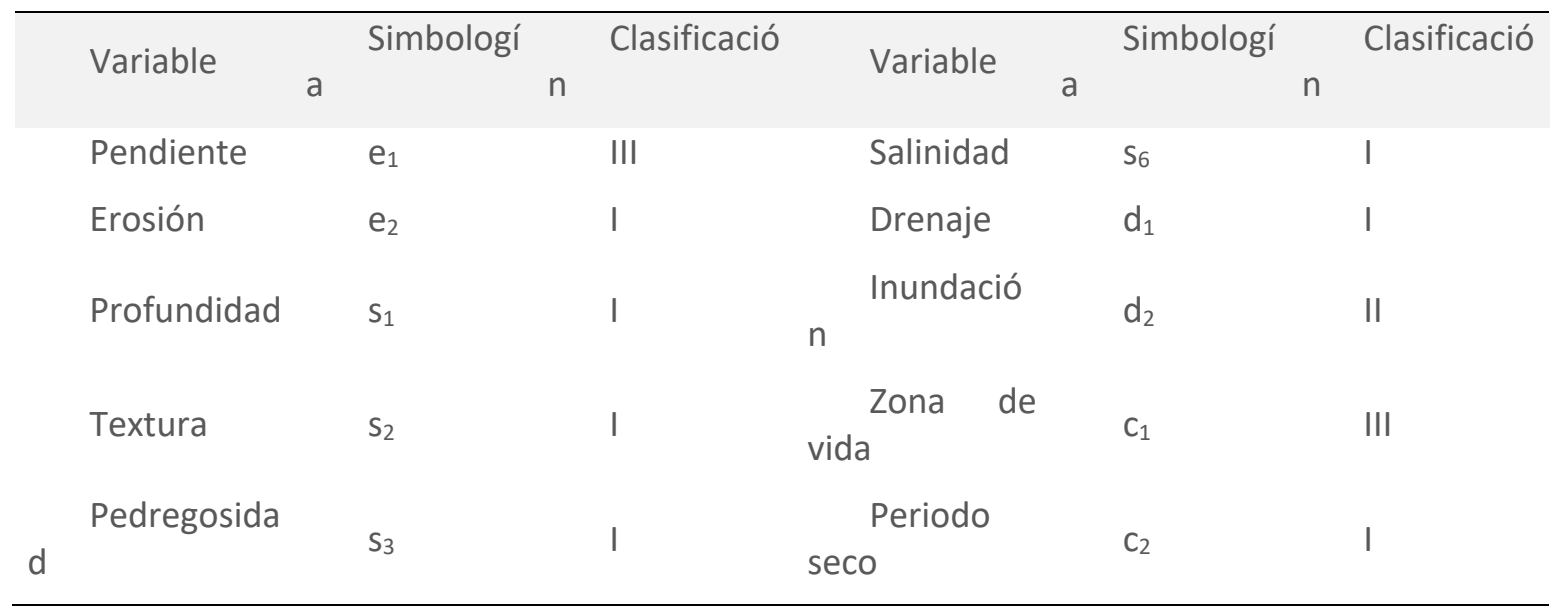




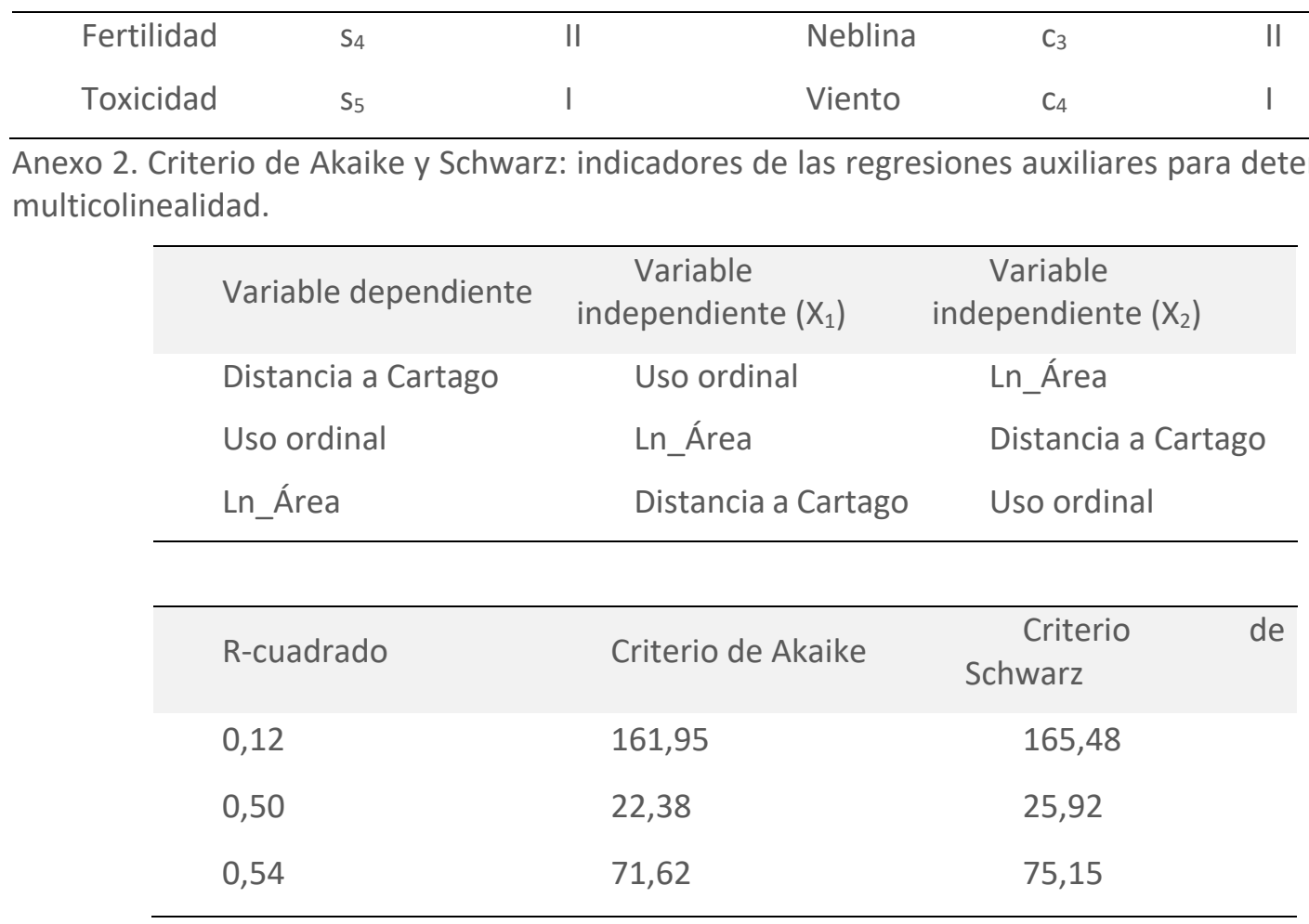

Anexo 3. Contraste de heterocedasticidad de Breusch-Pagan.

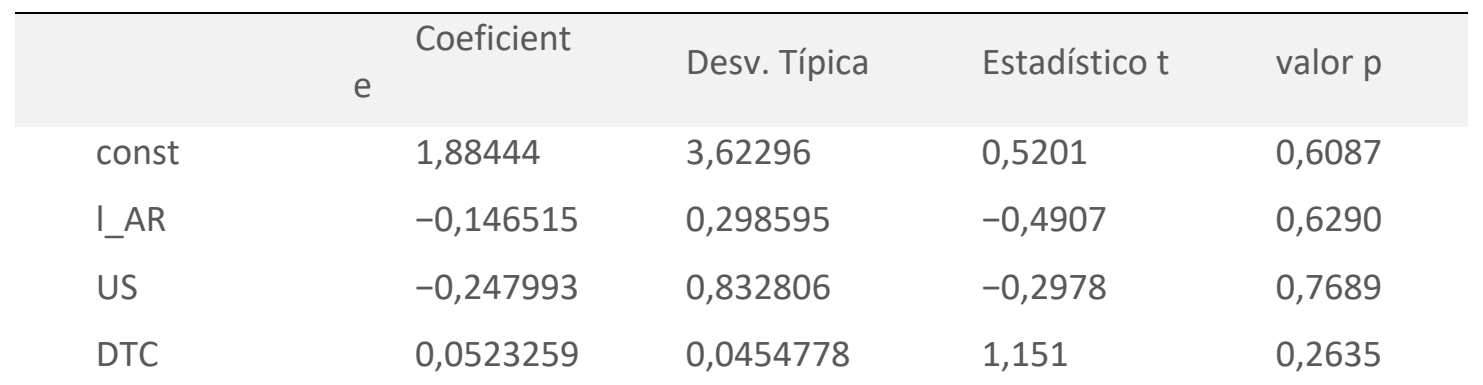

Suma de cuadrados explicada $=2,6205$

Estadístico de contraste: $\mathrm{LM}=1,310250$, con valor $\mathrm{p}=\mathrm{P}($ Chi-cuadrado $(3)>1,310250)=$ 0,726701

Anexo 4. Contraste Breusch-Godfrey de autocorrelación hasta el orden 3.

\begin{tabular}{|c|c|c|c|c|}
\hline & Coeficiente & $\begin{array}{l}\text { Desv. } \\
\text { Típica }\end{array}$ & $\begin{array}{l}\text { Estadístico } \\
\mathrm{t}\end{array}$ & valor $p$ \\
\hline const & 0,362032 & 1,19198 & 0,3037 & 0,7650 \\
\hline I_AR & $-0,0106771$ & 0,0923903 & $-0,1156$ & 0,9094 \\
\hline US & $-0,115607$ & 0,277949 & $-0,4159$ & 0,6827 \\
\hline DTC & $-0,00635676$ & 0,0142981 & $-0,4446$ & 0,6622 \\
\hline uhat_1 & 0,394737 & 0,252356 & 1,564 & 0,1362 \\
\hline uhat_2 & $-0,174679$ & 0,256650 & $-0,6806$ & 0,5053 \\
\hline
\end{tabular}




\begin{tabular}{|c|c|c|c|c|}
\hline uhat_3 & $-0,0685418$ & 0,292757 & $-0,2341$ & 0,8177 \\
\hline \multicolumn{5}{|c|}{ R-cuadrado $=0,133133$} \\
\hline \multicolumn{5}{|c|}{$\begin{array}{l}\text { Estadístico de contraste: } L M F=0,870281 \text {, con valor } p=P(F(3,17)>0,870281)= \\
76\end{array}$} \\
\hline \multicolumn{5}{|c|}{$\begin{array}{l}\text { Estadístico alternativo: } \mathrm{TR}^{\wedge} 2=3,195184 \text {, con valor } \mathrm{p}=\mathrm{P}(\text { Chi-cuadrado }(3)>3,19518) \\
, 362\end{array}$} \\
\hline
\end{tabular}

Anexo 5. Referencias y características de los terrenos para el método comparativo.

\begin{tabular}{|c|c|c|c|c|c|c|}
\hline & Variables & Área (m) & $\begin{array}{l}\text { Distancia } \\
(\mathrm{km})\end{array}$ & Uso & Acceso & $\begin{array}{l}\text { Precio } \\
\left(\mathbb{H} / \mathrm{m}^{2}\right)\end{array}$ \\
\hline & Fórmula & Potencial & Exponencial & Potencial & Exponencial & - \\
\hline & Avalúo & $7.665,75$ & 11,5 & 5 & 9 & - \\
\hline 1 & Referencia & 5.000 & 10,8 & 4 & 4 & 11.000 \\
\hline 2 & Referencia & 120.000 & 12,3 & 5 & 6 & 3.827 \\
\hline 3 & Referencia & 70.000 & 14,7 & 5 & 9 & 3.929 \\
\hline 4 & Referencia & 13.000 & 16,4 & 4 & 4 & 8.000 \\
\hline 5 & Referencia & 43.000 & 19,2 & 5 & 6 & 3.057 \\
\hline 6 & Referencia & 182.000 & 19,3 & 5 & 6 & 2.714 \\
\hline
\end{tabular}

Anexo 6. Factores de homologación para el método comparativo.

\begin{tabular}{|c|c|c|c|c|c|c|}
\hline Variables & $(\mathrm{m})^{\text {Área }}$ & $\begin{array}{l}\text { Distanc } \\
\text { ia }(\mathrm{km})\end{array}$ & Uso & Acces & $\begin{array}{l}\text { Factor } \\
\text { de } \\
\text { homologació } \\
n\end{array}$ & $\begin{array}{l}\text { Valor } \\
\text { homologado } \\
\left(\$ / \mathrm{m}^{2}\right)\end{array}$ \\
\hline ia 1 Referenc & $3^{0,910}$ & 0,9589 & $1^{0,791}$ & $3^{0,717}$ & 0,4953 & 5.448 \\
\hline ia $2^{\text {Referenc }}$ & $\begin{array}{ll} & 1,831 \\
5 & \end{array}$ & 1,0492 & $\begin{array}{ll}1,000 \\
0\end{array}$ & 0,819 & 1,5743 & 6.024 \\
\hline ia $3^{\text {Referenc }}$ & $\begin{array}{ll} & 1,626 \\
7 & \end{array}$ & 1,2117 & $\begin{array}{ll} & 1,000 \\
0 & \end{array}$ & $\begin{array}{ll} & 1,000 \\
0 & \end{array}$ & 1,9711 & 7.744 \\
\hline ia 4 Referenc & $2^{1,123}$ & 1,3418 & $1^{0,791}$ & $3^{0,717}$ & 0,8552 & 6.842 \\
\hline
\end{tabular}




\begin{tabular}{|c|c|c|c|c|c|c|}
\hline ia 5 Referenc & $4^{1,461}$ & 1,5872 & $\begin{array}{ll}1,000 \\
0\end{array}$ & $2^{0,819}$ & 1,9003 & 5.809 \\
\hline ia 6 Referenc & $3 \begin{array}{ll}2,007 \\
3\end{array}$ & 1,5968 & $0^{1,000}$ & $2^{0,819}$ & 2,6259 & 7.127 \\
\hline & & & & $\begin{array}{l}\text { Valor } \\
\left(\mathbb{\$} / \mathrm{m}^{2}\right)\end{array}$ & promedio & 6.499 \\
\hline & & & & \multicolumn{2}{|c|}{ Valor total $(\mathbb{W})$} & $43 \quad 49.819 .5$ \\
\hline
\end{tabular}

Anexo 7. Estado de Flujo Neto de Efectivo para el cultivo de papa.

\begin{tabular}{ll}
\hline Rubro & Año $1(\mathbb{W})$ \\
Ingresos & 18.299 .120 \\
(-) Costos de producción & 11.969 .814 \\
Utilidad Bruta & 6.329 .306 \\
(-) Gastos de ventas & 914.956 \\
(-) Gastos administrativos & 1.196 .981 \\
(-) Depreciación & 120.000 \\
Utilidad Antes de & 4.097 .369 \\
Impuestos denta & 409.737 \\
(-) Impuesto de & \\
(10\%) & 3.687 .632 \\
Utilidad Neta & 120.000 \\
Depreciación & 3.807 .632 \\
Flujo Neto de Efectivo
\end{tabular}

Anexo 8. Materiales, cantidades y costo del invernadero presente en la propiedad.

\begin{tabular}{|c|c|c|c|c|}
\hline Detalle & Cantidad & Unidad & $\begin{array}{c}\text { Costo } \\
\text { unitario }(\mathbb{W})\end{array}$ & $(\mathbb{W})$ Costo total \\
\hline Plástico invern. dripblock & 1455 & $\mathrm{~m} 2$ & 553 & 803.888 \\
\hline Malla antitrips $40 / 25$ & 438 & $\mathrm{~m} 2$ & 611 & 267.667 \\
\hline Cemento & 9 & Saco & 6.400 & 57.600 \\
\hline Arena & 1 & $\mathrm{~m} 2$ & 16.070 & 16.070 \\
\hline Piedra & 2 & $\mathrm{~m} 2$ & 17.400 & 34.801 \\
\hline Canaleta prensa & 360 & $2 \mathrm{mts}$ & 1.685 & 606.772 \\
\hline Resorte para prensa & 32 & $\mathrm{Kg}$ & 2.569 & 82.204 \\
\hline Tubo 2 1/2" 1,8mm & 113 & $6 \mathrm{mts}$ & 16.208 & 1.831 .504 \\
\hline Tubo 1" 1,5mm & 136 & $6 \mathrm{mts}$ & 7.360 & 1.001 .880 \\
\hline
\end{tabular}




\begin{tabular}{|l|l|l|l|l|}
\hline Tubo 2" 1,5mm & 45 & $6 \mathrm{mts}$ & 13.624 & 613.080 \\
\hline Soldadura Hilco 6013 & 14 & Kg & 4.525 & 63.350 \\
\hline Tornillos & 1000 & unit & 33 & 33.170 \\
\hline \multirow{2}{*}{} & \multicolumn{2}{|c|}{ Total } & 5.411 .985 \\
\cline { 3 - 5 } & \multicolumn{2}{|c|}{ Con Transporte } & 5.736 .704 \\
\cline { 3 - 5 } & \multicolumn{2}{|c|}{ Con M.O. } & 10.326 .068 \\
\cline { 3 - 5 }
\end{tabular}

Anexo 9. Diseño preliminar del plano para la cabaña.

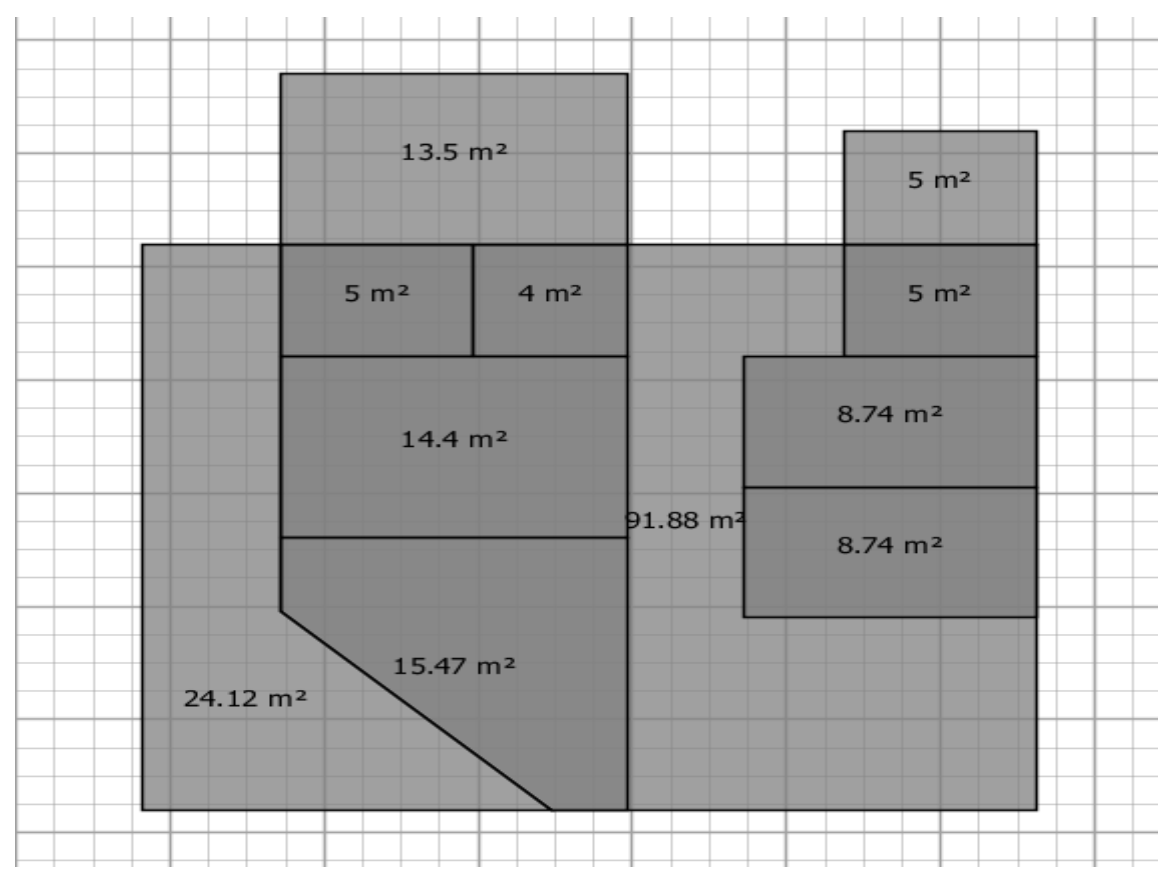

Anexo 10. Diseño preliminar del plano para el granero. 


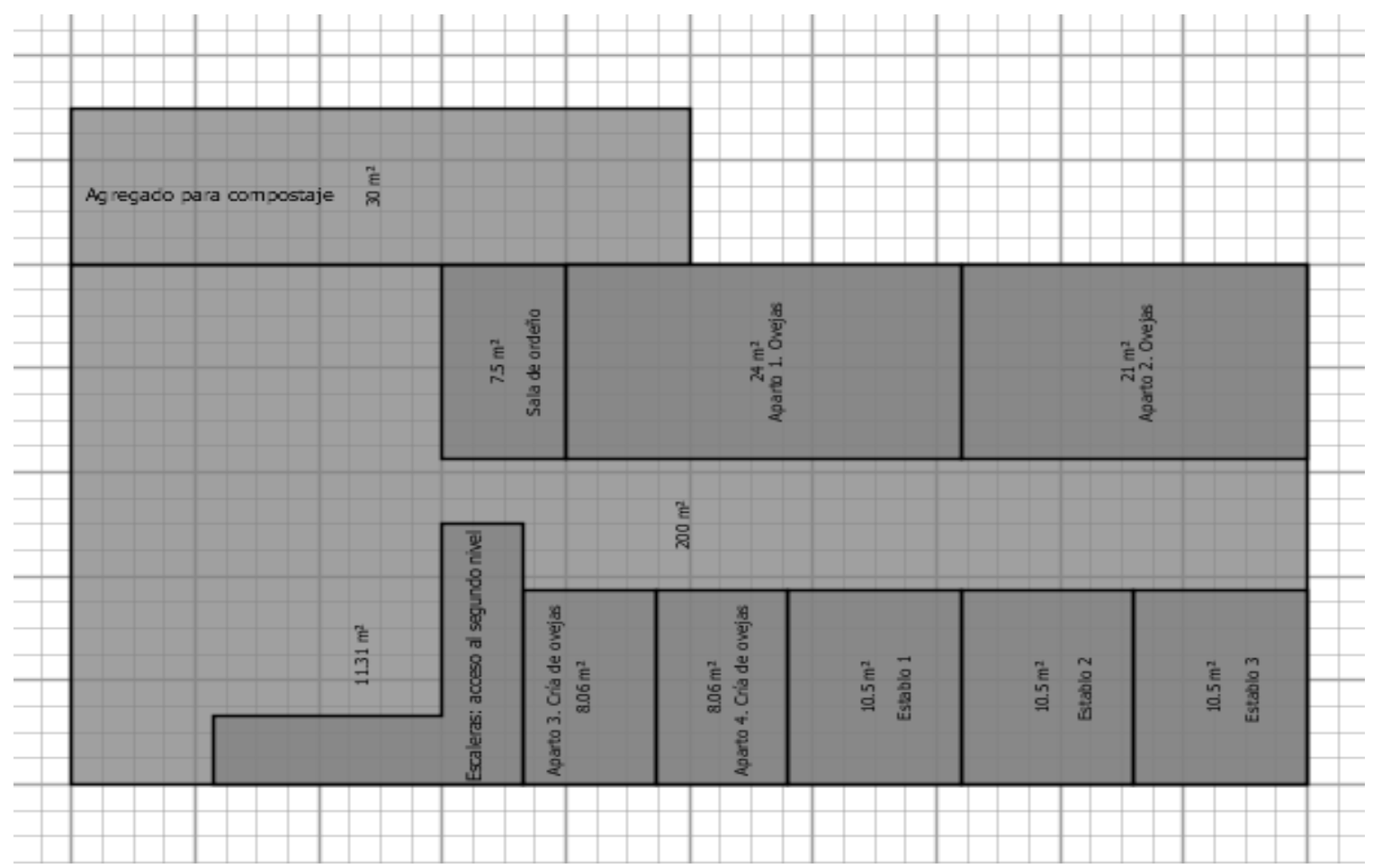

